\title{
DRA. LARA: ASSISTENTE VIRTUAL DE APOIO E ACOMPANHAMENTO AO PRÉ-NATAL
}

\author{
Laryssa Carvalho, Marianne Albuquerque, Rodrigo Nogueira, Johannes Lochter \\ Departamento de Engenharia de Computação, \\ Centro Universitário Facens, Brasil
}

\begin{abstract}
RESUMO
Aproximadamente, 830 mulheres morrem por dia por causas evitáveis relacionadas à gestação, e apesar dos esforços para proporcionar acesso universal e de qualidade à saúde reprodutiva, esse problema ainda é um desafio em aberto. Este trabalho propõe utilizar um aplicativo para fazer o acompanhamento pré-natal como apoio ao médico da gestante para lembrá-la dos exames e responder perguntas frequentes que as gestantes têm. O mecanismo para tornar essa solução possível é um chatbot que processa as perguntas e as intenções da gestante com inteligência artificial e encontra respostas em uma base de conhecimento. As contribuições são a base de conhecimento curada e a implementação preliminar.
\end{abstract}

\section{PALAVRAS-CHAVE}

Pré-Natal, Chatbot, E-Health, Processamento de Linguagem Natural, Inteligência Artificial

\section{INTRODUÇÃO}

O pré-natal é o acompanhamento na área da medicina prestado à mulher durante o período da gestação com o objetivo de garantir a saúde da mãe e do bebê. Sendo assim, essa assistência tem papel fundamental na prevenção e detecção precoce de doenças que podem acometer tanto a gestante como o feto, reduzindo assim o risco de natimortos e de complicações durante a gravidez. Estima-se que, em 2015, 303 mil mulheres morreram por causas relacionadas à gravidez; 2,7 milhões de bebês morreram durante os 28 primeiros dias de vida; e 2,6 milhões de bebês eram natimortos (OMS, 2018).

A mesma pesquisa da Organização Mundial de Saúde (2018) aponta que, aproximadamente, 830 mulheres morrem por dia por causas evitáveis relacionadas à gestação e, embora esse número pareça alarmante, o número já foi mais expressivo e diminuiu em $40 \%$ entre 1990 e 2015.

Muitos esforços vêm sendo implementados mundialmente com o intuito de proporcionar acesso universal e de qualidade com atenção à saúde reprodutiva. No país, a cobertura pré-natal aumentou significativamente após a adoção do Programa Nacional Humanização do Pré-Natal e Nascimento, cuja principal estratégia é assegurar a melhoria do acesso, cobertura e qualidade da assistência ao pré-natal e parto (Rosa, 2014).

Com o avanço das tecnologias e a crescente adoção das pessoas na utilização de smartphones, este artigo propõe o emprego de assistentes virtuais (ou chatbots) como agentes conversacionais para incentivar e alertar as mulheres com relação à importância do pré-natal, assim como auxiliá-las durante esse período da gestação sanando as suas principais dúvidas. Com o uso de técnicas de processamento de linguagem natural e aprendizado de máquina, por meio de um aplicativo para dispositivos móveis, qualquer gestante poderá ter acesso à assistente inteligente Dra. Lara para conversar e ter ajuda durante essa etapa importante de suas vidas.

\section{TRABALHOS RELACIONADOS}

Com o avanço da tecnologia, os smartphones se tornaram um item imprescindível para a maioria da população e junto com eles vieram os aplicativos. Eles têm o intuito de facilitar cada vez mais a vida das pessoas desde tarefas simples do dia-a-dia, como a troca de mensagens até tarefas mais complexas, como compras online (Dino, 2018). 
A tecnologia de chatbot tem sido usada para diferentes fins nas mais diversas áreas. Dentro da saúde e medicina voltada para mulheres é possível citar alguns exemplos, como os produtos Safedrugbot e Izzy. O chatbot Safedrugbot é voltado para a correta orientação sobre quais medicamentos a lactante pode utilizar, enquanto o chatbot Izzy auxilia a lembrar e identificar os períodos férteis, além de fornecer informações sobre ciclos menstruais e problemas sexuais.

Alguns outros produtos atuam de maneira híbrida oferecendo o suporte por texto para situações mais simples e problemas menos complexos, mas também oferecem a possibilidade de consulta com médicos reais, como Babylon Health e Gyant.

Apesar de serem inúmeras as soluções e propostas dentro da área da medicina e saúde que envolvem a aplicação de inteligência artificial para associar as necessidades dos pacientes com bases de conhecimentos médicos, buscas extensas conduzidas pelos autores não resultaram em nenhum tipo de chatbot que atuasse de forma ativa com os usuários, apenas de forma passiva. Os chatbots ficam disponíveis para responder perguntas e associar sintomas à doenças, mas não fazem perguntas, lembretes e nem tomam iniciativa de questionar o usuário. Esse trabalho apresenta um chatbot com características ativas, além dos recursos passivos implementados em outros produtos e soluções.

No intervalo de desenvolvimento desse trabalho, uma solução interessante encontrada foi a PROMPTS, que funciona a partir de mensagens de texto e foi capaz de atender mais de 11 mil mulheres em situações de risco no Quênia. Esse trabalho é o mais relevante e o mais próximo à proposta deste artigo e questiona a quantidade de esforço de processamento de linguagem natural que deve ser aplicada para entender as mensagens das gestantes, indo além de utilizar um conjunto de regras ou palavras-chave nas mensagens, estratégias empregadas por soluções voltadas para gestantes, tais como Nivi, Pregnancy Bot, Sophie Bot, Lily.Health e Ada.

Em chatbots, o processamento de linguagem natural juntamente com o aprendizado de máquina é utilizado para treinar o modelo, tornando-o capaz de entender as intenções do usuário e as entidades, que são as partes mais relevantes do texto. Além disso, essa combinação de tecnologias é responsável por treinar o modelo para saber qual ação deve ser tomada durante o fluxo da conversa (Kowatsch, 2017).

Neste trabalho, a proposta da Dra. Lara utiliza o framework Rasa (Bocklisch, 2017) para compreensão de linguagem natural e utiliza técnicas consideradas estado-da-arte como representação distribuída e vetorial, além de redes neurais recorrentes (Mikolov, 2010; Tai, 2015) para compreender as mensagens de texto sem depender de conjuntos de regras fixas ou sistemas léxicos (Wang \& Manning, 2012).

\section{DESENVOLVIMENTO}

O desenvolvimento do chatbot Dra. Lara foi conduzido com acompanhamento de profissionais médicos para validar toda a base de conhecimento sobre gestação. Além disso, foi estudado o protocolo do Sistema Único de Saúde do Brasil para saber quais exames são recomendados de fazer conforme os diferentes estágios da gravidez (Tabela 1).

De maneira ativa, o chatbot Dra. Lara questionará a usuária sobre as datas dos exames e atendimentos, armazenando para o cadastro do usuário quais requisitos foram cumpridos. $\mathrm{Na}$ falta do cumprimento ou pendência, o chatbot voltará a lembrar o usuário em intervalos configurados sobre as necessidades e importâncias de realizar todas as exigências da cartilha.

Tabela 1. Relação de exames necessários durante a gestação

\begin{tabular}{ll}
\hline Quando deve ser feito & Exame \\
\hline Assim que inicia o pré-natal & Sangue \\
& Urina \\
& Fezes \\
& Ultrassonografia intravaginal \\
\hline $1^{\circ}$ Trimestre & Ultrassonografia de transluscência nucal \\
\hline $2^{\circ}$ Trimestre & Triagem de diabetes gestacional \\
\hline $3^{\circ}$ Trimestre & Triagem de estreptococo beta-hemolítico \\
& Ultrassonografia do terceiro semestre \\
\hline
\end{tabular}


Uma coleção de 243 perguntas em português curadas por profissionais médicos também foi incluída na base de conhecimento do chatbot Dra. Lara para ter capacidade de responder às perguntas comuns que as gestantes normalmente querem saber, como a possibilidade de comer um determinado elemento ou riscos associados à prática de um determinado exercício. A coleção de perguntas é pública e está disponível em https://github.com/marikell/dralaradados/blob/master/perguntas-respostas.csv.

O chatbot foi preparado para conduzir rapidamente um questionário com novas usuárias de modo a coletar e entender em qual estágio da gestação a gestante está. Em seguida, a gestante pode fazer as perguntas que tiver curiosidade em ter resposta e, esporadicamente, o chatbot poderá perguntar e lembrar a usuária sobre exames, consultas e cuidados básicos de saúde.

Para tornar o funcionamento do chatbot possível foi planejada uma plataforma (Figura 1) consistindo de duas partes principais: front-end (cliente) e back-end (servidor). A linguagem de programação Python subsidia toda a implementação de servidor e comunicação entre o aplicativo no smartphone do usuário e o servidor onde os dados são armazenados e manipulados. Todo o sistema está organizado em contêineres do Docker para tornar a distribuição e o deploy mais simples.

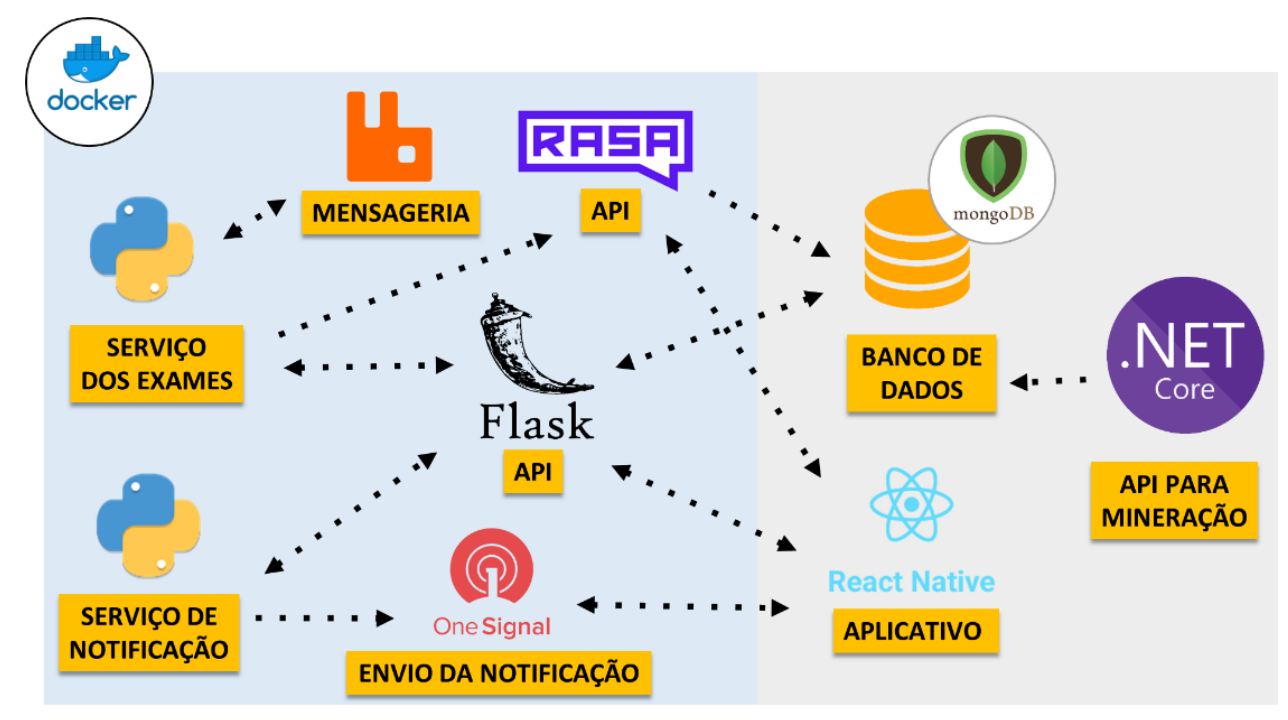

Figura 1. Diagrama da plataforma do chatbot Dra. Lara

A interface do aplicativo foi feita em React Native e algumas telas são apresentadas na Figura 2, na ordem: tela de conversação, tela de checklist dos exames nos períodos corretos da gravidez e tela de notificações com sugestões e dicas para a gestante usuária. O repositório com o código do desenvolvimento preliminar está disponível em https://github.com/marikell/eve-platform.

\section{CONCLUSÃO PRELIMINARES E PRÓXIMOS PASSOS}

O presente artigo traz a proposta de tratar o problema da mortalidade no pré-natal de causas tratáveis que acomete gestantes ao redor do mundo. O mecanismo proposto envolve a utilização de um aplicativo com capacidade de processamento de linguagem natural para entender as perguntas das gestantes e entregar respostas adequadas a partir de uma base de dados curada por profissionais médicos e da saúde.

Os próximos passos são tornar o aplicativo público nas principais lojas de aplicativos e coletar as informações para ser possível realizar a mineração de dados e tornar os dados públicos, porém anônimos, para mineração da gestão pública de saúde através de uma API. 


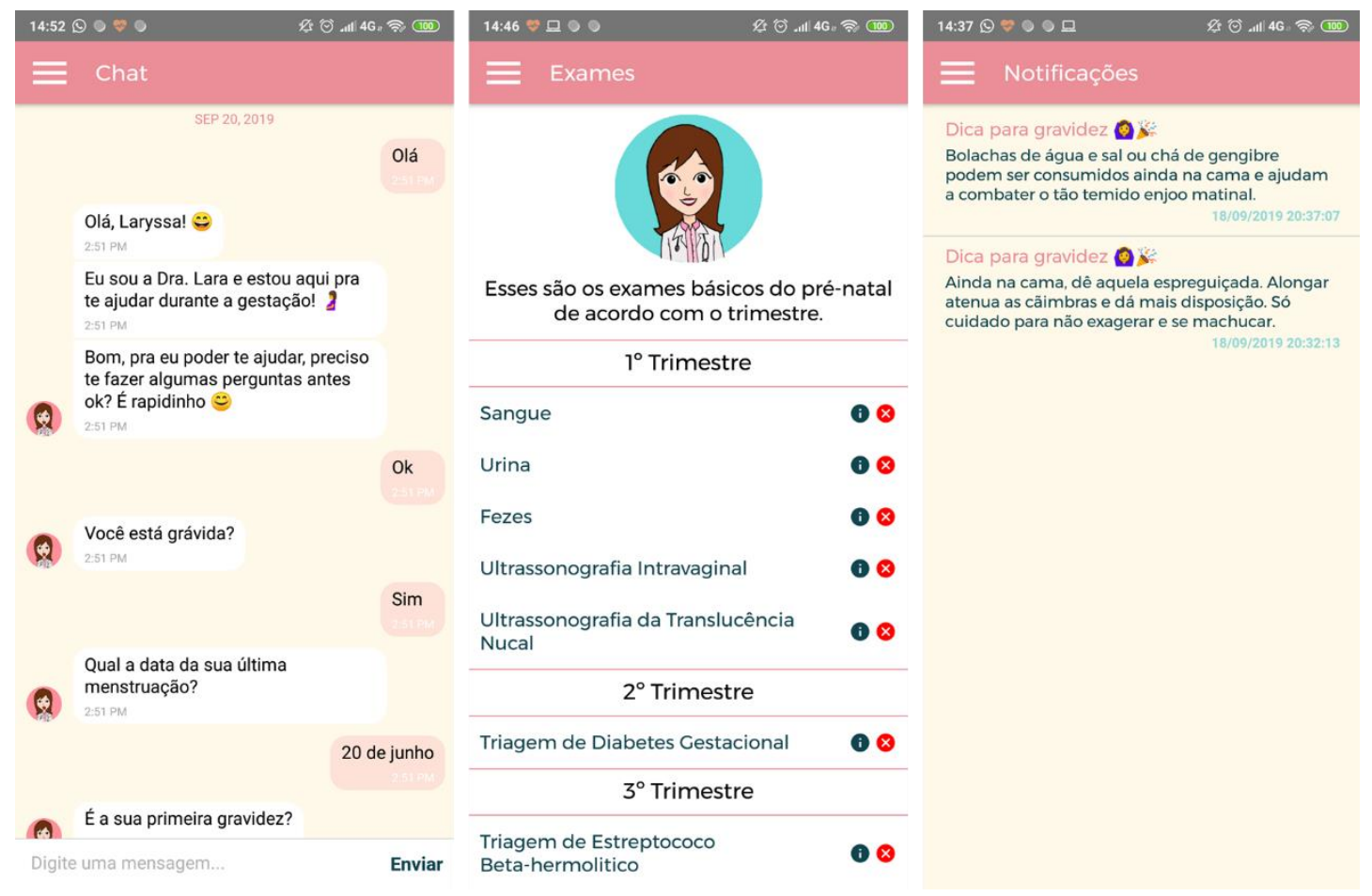

Figura 2. Algumas telas desenvolvidas do aplicativo e chatbot Dra. Lara

\section{REFERÊNCIAS}

Bocklisch, T. et al., 2017. Rasa: Open Source Language Understanding and Dialogue Management. In arXiv, preprint arXiv:1712.05181.

DINO, 2018. Avanço dos aplicativos no Brasil estimula startups e grandes empresas, acessado em 20 Setembro 2019, <https://exame.abril.com.br/negocios/dino/avanco-dos-aplicativos-no-brasil-estimula-startups-e-grandes-empresas>.

Kowatsch, T. et al, 2017. Text-based Healthcare Chatbots Supporting Patient and Health Professional Teams: Preliminary Results of a Randomized Controlled Trial on Childhood Obesity. In Persuasive Embodied Agents for Behavior Change (PEACH 2017) Workshop, 17th International Conference on Intelligent Virtual Agents (IVA 2017), Stockholm, Sweden.

Mikolov, T. et al, 2010. Recurrent neural network based language model. In Interspeech, Vol. 2, No. 1, pp 3-7.

OMS, 2018. Folha informativa - Mortalidade materna, acessado em 20 Setembro 2019, $<$ https://www.paho.org/bra/index.php?option=com_content\&view=article\&id=5741:folha-informativa-mortalidadematerna\&Itemid $=820>$.

ROSA, C. Q. et al, 2014. Fatores associados à não realização de pré-natal em município de grande porte, In Revista de Saúde Pública, Vol. 48, No. 6, pp.977-984.

Tai, K. S. et al, 2015. Improved semantic representations from treestructured long short-term memory networks. In arXiv, preprint arXiv:1503.00075.

Wang, S. \& Manning, C. D., 2012. Baselines and bigrams: Simple, good sentiment and topic classification. In Proceedings of the 50th Annual Meeting of the Association for Computational Linguistics: Short Papers, Vol. 2, No. 1, pp 90-94. 